\title{
Une culture d'équations et de codes ? le metteur-en-code, entre la recherche en mécanique numérique et l'industrie aéronautique
}

\section{Résumé :}

L'article s'interroge sur la base phénoménologique qui permet les échanges entre un laboratoire de mécanique et l'industrie aéronautique dans le domaine de la mécanique informatisée, c'està-dire la recherche des structures qui gouvernent les relations entre les différentes catégories d'acteurs et qui donnent un sens à leurs pratiques. Pour les appréhender, nous allons étudier le partage, la constitution et la reformulation permanente de la Méthode des Eléments Finis (MEF) comme base du travail commune aux deux mondes. La MEF est la méthode mécanique/numérique qui a permis le développement de logiciels de calcul pour l'industrie. L'article s'interroge ainsi sur les conditions de possibilité du lien entre le laboratoire et les entreprises du secteur aéronautique sur la base de la transformation de la mécanique, suite à la généralisation des méthodes numériques dans les sciences et les pratiques des ingénieurs. Nous allons aborder les différentes transformations que les ordinateurs introduisent dans la mécanique, dans le travail des ingénieurs et les pratiques de recherche, jusqu'à la consolidation de la culture d'une mécanique informatisée qui constitue les bases sur lesquelles se construit le lien entre notre laboratoire et l'industrie aéronautique qui fait naître le metteur-en-code.

\section{Introduction, le lien recherche-Industrie à travers le concept de culture}

Depuis les années 1970, l'aéronautique et les sciences de l'ingénieur se transforment grâce au développement de l'informatique (Carpentier, 2010). Ces transformations s'accompagnent de la constitution d'un espace commun de communication entre l'industrie et la recherche. Ce monde complexe entre la recherche en mécanique numérique et l'industrie constitue une sorte de culture commune que le metteur-en-code incarne. Le metteur-en-code est un acteur qui apparaît grâce à ces transformations entre l'ingénieur et le chercheur.

Cet article $^{1} \mathrm{~s}^{\prime}$ interroge sur la base phénoménologique qui permet les échanges entre un laboratoire de mécanique et l'industrie aéronautique dans le domaine de la mécanique

\footnotetext{
${ }^{1}$ L'article est le résultat d'un travail ethnographique au sein d'un laboratoire de mécanique située dans la banlieue sud de Paris. Le corpus mobilisé se compose d'entretiens semi-directifs réalisés pendant le
} 
informatisée, c'est-à-dire la recherche des structures qui gouvernent les relations entre les différentes catégories d'acteurs et qui donnent un sens à leurs pratiques. Pour les appréhender, nous allons étudier le partage, la constitution et la reformulation permanente de la Méthode des Eléments Finis (MEF) comme base du travail commune aux deux mondes. La MEF est la méthode mécanique/numérique qui a permis le développement de logiciels de calcul pour l'industrie. L'article s'interroge ainsi sur les conditions de possibilité du lien entre le laboratoire et les entreprises du secteur aéronautique sur la base de la transformation de la mécanique, suite à la généralisation des méthodes numériques dans les sciences et les pratiques des ingénieurs. Nous allons aborder les différentes transformations que les ordinateurs introduisent dans la mécanique, dans le travail des ingénieurs et les pratiques de recherche, jusqu'à la consolidation de la culture d'une mécanique informatisée qui constitue les bases sur lesquelles se construit le lien entre notre laboratoire et l'industrie aéronautique qui fait naître le metteur-en-code.

Le laboratoire de mécanique analysé se distingue par ses travaux autour du calcul numérique. Cette orientation a contribué au renforcement des relations entre le laboratoire et l'industrie aéronautique. Le laboratoire et l'industrie aéronautique produisent un espace d'échange commun et plus largement une culture commune orientés vers la compréhension de phénomènes de la mécanique, notamment l'endommagement des matériaux composites.

Nous entendons par culture l'ensemble des pratiques, des artefacts et des symboles créés par des hommes et des femmes et à leur disposition, qui constituent un terreau de sens pour les collectifs. Corrélativement, ces pratiques, artefacts et symboles témoignent des modes dans lesquels une culture produite du sens et un ordre social et matériel. La culture est constituée par l'ensemble des systèmes sociaux de traitement de l'information (Cicourel, 2011 : 340 ; Roberts, 1964). La particularité de l'information n'est pas son contenu, mais le fait de circuler entre les individus et les objets du monde ${ }^{2}$. La culture commune au laboratoire et à l'industrie

travail de terrain avec des chercheurs, des metteurs-en-code et des ingénieurs du secteur aéronautique français.

${ }^{2}$ C'est pour cela que les cognitivistes vont parler de cognition distribuée (Hutchins, 1995 ; Heintz, 2011). La complexité des systèmes de traitement de l'information qui font la culture dépasse l'expérience individuelle. Mais la question de Roberts est quel type de traitement de l'information constitue une culture ? Il dira qu'une culture reçoit de l'information, la stocke, développe des moyens pour la récupérer, la transmettre, la mette à disposition des membres, la transformer et même l'éliminer. La culture fonctionne comme une sorte d'économie informationnelle ou l'information est stockée dans l'esprit des individus et dans les artéfacts (Roberts, 1964). Le monde objectif est intégré dans l'expérience subjective. Le sujet n'existe pas en dehors de relations objectives dans lesquelles il s'inscrit, c'est-à-dire en dehors d'un système de cognition distribué. Pour l'étude de la science, les constructivistes vont partir de l'idée que l'information est créée et stockée dans les artéfacts et les liens qui se tissent à l'intérieur des murs des laboratoires et par rapport aux agents extérieurs. L'objectif des constructivistes sera de comprendre la façon dont les ressources informationnelles propres à la science forment des cultures particulières qui produisent des effets sur l'ensemble de la société. La culture inclut ainsi l'ensemble des pratiques, des artéfacts et des symboles à 
aéronautique se constitue autour de la MEF et se distingue par les modes de traitement de l'information, les méthodes, les pratiques des acteurs, ainsi que par le type d'objets que ces acteurs manipulent. Cette méthode propose un système de symboles et fonctionne comme un langage partagé entre le laboratoire et les entreprises.

L'article montre la manière dont s'organisent les relations entre le laboratoire et l'industrie dans cet espace commun et comment se configure une culture fortement liée au numérique au sein de laquelle apparaît le metteur-en-code.

\section{La MEF, un langage commun à la recherche et à l'aéronautique autour des problématiques de la mécanique numérique}

\section{Principe et histoire des Eléments Finis (EF)}

Les EF sont apparue très rapidement dans mon travail de terrain. A chaque discussion sur leur travail avec les industriels, les metteurs-en-code me parlaient de cette méthode avec naturel et assurance. Quand ils se rappelaient mon appartenance disciplinaire, ils synthétisaient « tu sais... tu prends ta pièce, tu la discrétises, tu choisis ton matériau et tu lances ton calcul » (Marie, Metteure-en-code). Ce type de déclarations très technique recèle pourtant un sens sociologique. La MEF s'est avéré être à la base de la communication entre les chercheurs et les ingénieurs. Or, si la méthode permettait la communication, les chercheurs et les ingénieurs révélaient des modes d'appropriations et des intérêts différents à son égard. En outre, la méthode sert de base pour les logiciels de simulation numérique qui, dans le discours des chercheurs et des ingénieurs (des entreprises), se révélait être une dimension très importante de leur travail et dans leurs échanges.

La MEF est une méthode numérique qui permet de trouver une solution approchée à un système d'équations qui décrivent le comportement d'un objet, matériau ou structure. Elle est basée sur le principe de discrétisation de la géométrie qu'on veut simuler, par exemple l'aile d'un avion, un fuselage, une pièce etc. Ainsi, techniquement, la MEF consiste à découper la pièce à calculer en des petits morceaux, dans un environnement de calcul numérique.

\footnotetext{
disposition des hommes et des femmes qui constituent le terreau de sens pour les collectivités. Corrélativement, ces pratiques, artéfacts et symboles témoignent des modes dans lesquels une culture produite du sens ou produit un ordre du monde.
} 
Le mésomodèle des matériaux composites est un modèle mécanique créé au sein du laboratoire qui représente le comportement d'un type de matériau très utilisé pour la fabrication d'avions, les matériaux composites. Le mésomodèle est le système d'équations qui décrit le comportement du composite. La MEF sera utilisée pour résoudre les équations du mésomodèle grâce aux logiciels de calcul industrielle. Les logiciels Abaqus et Samcef sont des logiciels EF car l'ingénieur peut y écrire des équations, à l'aide du mésomodèle s'il s'agit d'un matériau composite, dessiner la pièce, la discrétiser et résoudre les équations.

L’illustration 1 montre la hiérarchie des pratiques et des éléments constituant la mécanique numérique. Dans ce type de mécanique nous trouvons différents types des méthodes dont les EF. Sur la base des EF et à la frontière du laboratoire et des entreprises, les metteurs-en-code développent des logiciels de calcul numérique pour les ingénieurs des bureaux de méthodes industriels, notamment l'ingénieur-calcul dont nous parlons ultérieurement.

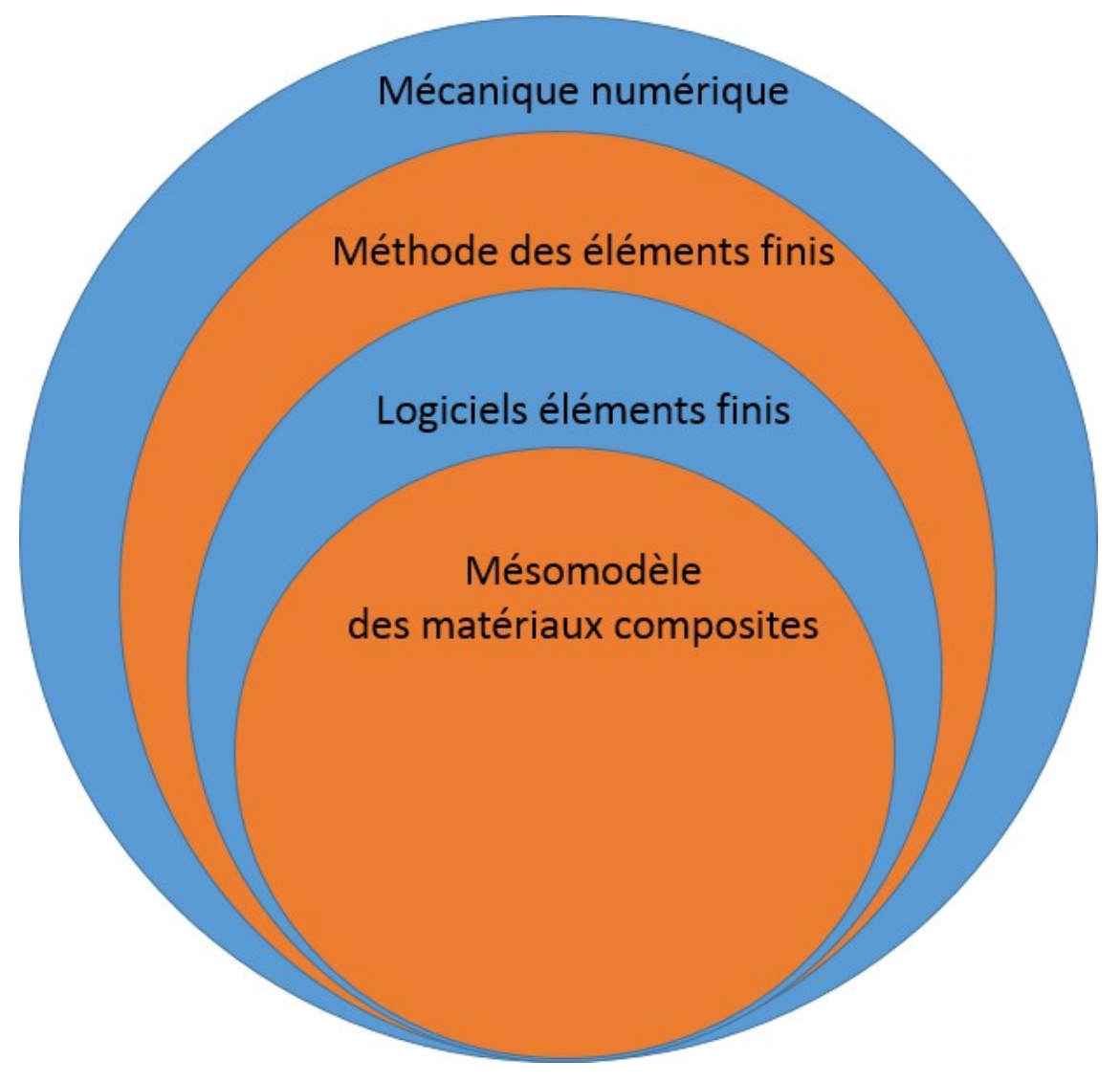

Illustration 1: Hiérarchie mécanique numérique, MEF et mésomodèle des matériaux composites

L'illustration 2 explique la relation entre la mécanique numérique, la MEF et les étapes des analyses selon cette méthode. 


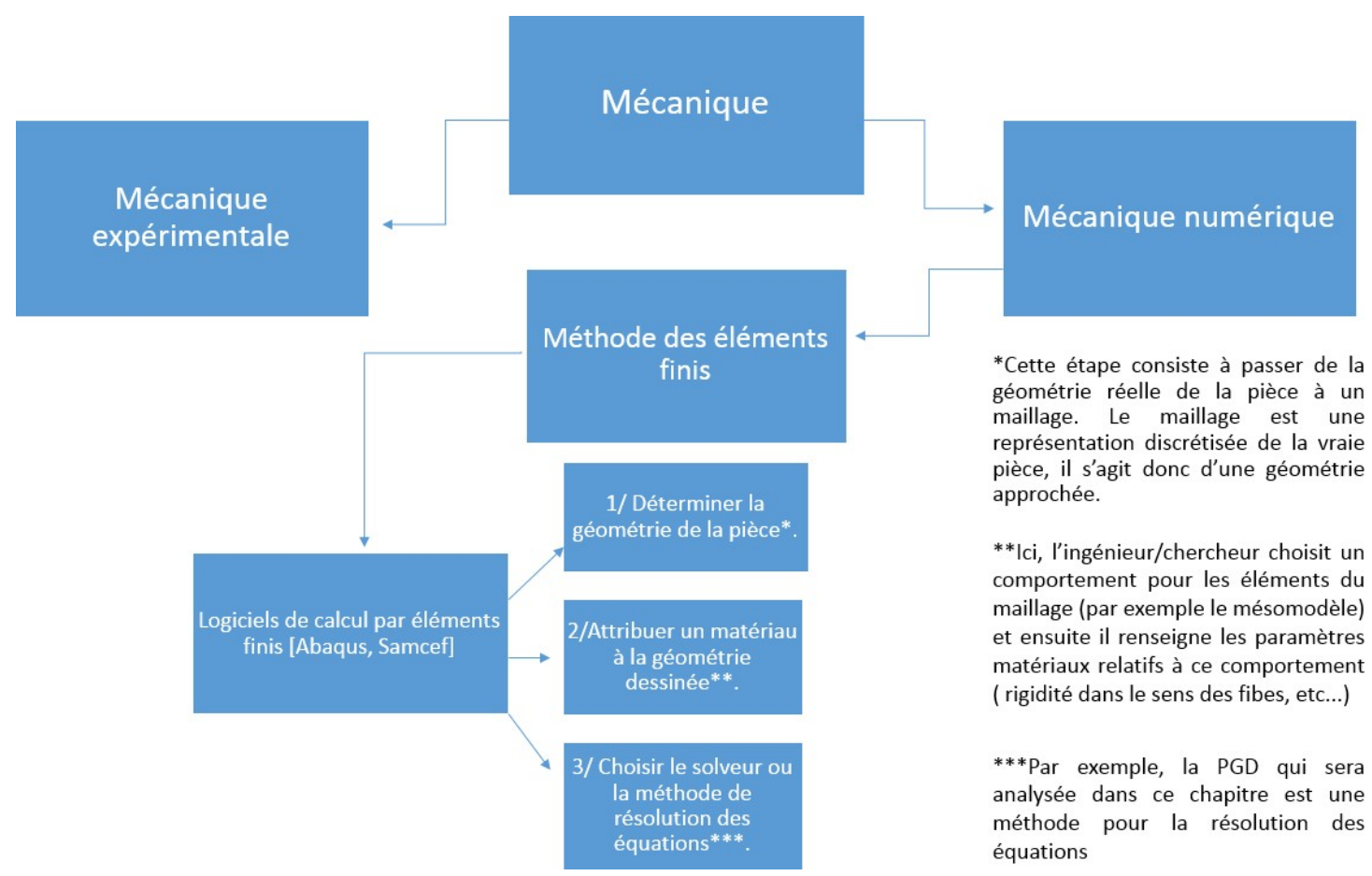

Illustration 2 : Mécanique numérique et MEF

Avec le développement de l'informatique dans les années soixante, la MEF a pu devenir un vrai outil d'ingénieur. En effet, la MEF est la partie théorique qui, avec le développement de l'informatique, a permis le développement des codes de calcul. Dans les années 1990, la méthode a complétement pénétré l'ingénierie au point de devenir la méthode presque universelle en mécanique des solides.

\section{Le laboratoire et la MEF}

Les méthodes numériques de calcul offrent des possibilités d'analyse de pièces à géométries complexes. Capables de prendre en compte des phénomènes mécaniques compliqués, elles occupent une place centrale dans les pratiques des ingénieurs.

Les ingénieurs calculent leurs objets et leurs systèmes sur la base des EF, et ces éléments leur permettent de limiter la complexité et ainsi de la maîtriser. Dans l'environnement de simulation, les ingénieurs pourront donc prendre en compte pour un calcul d'objet tout un ensemble de phénomènes qu'ils ne pouvaient pas intégrer avec les méthodes traditionnelles (analytiques) de la mécanique.

La MEF permet la communication et fonde l'espace de coopération entre le laboratoire et l'industrie. Néanmoins, les ingénieurs dans des entreprises et les chercheurs s'approprient de manière différente la méthode. Les ingénieurs vont exploiter les logiciels d'analyse par EF pour 
obtenir une simulation ou un objet numérique qui leur permettra d'avancer vers la fabrication des objets et des systèmes. La simulation par ordinateur permet la pré-création des objets techniques sous la forme d'objets numériques. Ces objets numériques sont conçus et testés selon les principes des méthodes comme celle des EF. Néanmoins, quand les ingénieurs projettent un objet technique sous la forme d'objet numérique, ils le font selon les méthodes fondamentalement développées dans la recherche mais aussi dans l'industrie du logiciel. La recherche du laboratoire étudié semble occuper une place intermédiaire entre la mécanique et l'informatique d'entreprise sans ne se confondre ni avec l'une ni avec l'autre. Les chercheurs, les metteurs-en-code et leurs interlocuteurs industriels dans l'aéronautique parlent la « même langue » et travaillent avec la langue de la simulation par EF mais leur appropriation et approche à la langue diffère sur certains points nodaux.

\section{L'ingénieur-calculs et les logiciels par EF, les exemples d'Abaqus et de Samcef}

La pratique du calcul par EF a fait apparaître un type d'ingénieur que Stephen Mer a appelé « ingénieur-calculs » (1999). Cet acteur de la production industrielle est chargé de la définition des caractéristiques et fonctionnelles du produit à l'aide de la simulation numérique. A partir des définitions des ingénieurs-calculs, les dessinateurs élaborent le plan technique des pièces et des objets, avant leur fabrication. Ainsi, nous reconnaissons une différence entre le travail des dessinateurs et le travail des ingénieurs-calculs. Ces derniers doivent définir les paramètres pour obtenir la performance optimale d'une pièce, un objet ou système, et cette performance dépend de ses besoins fonctionnels. Les paramètres de la performance optimale auront la « forme » des résultats des simulations. De ce fait, ils ont un lien direct avec les outils à travers lesquels ils ont été obtenus. Les logiciels « Eléments Finis » sont inséparables de la pratique des ingénieurscalculs. Parmi les logiciels les plus utilisés, nous pouvons citer : Abaqus de Dassault Systèmes, Castem du CEA, Code Aster d'EDF, Nastran de MSC Sofware Corp., Samcef de Samtech/Siemens.

Une fois que l'ingénieur-calcul a modélisé sa pièce, son objet ou son système, l'étape suivante sera de traduire les résultats de simulations en dessins techniques (Mer, 1999). L'ingénieurcalcul va évaluer différents matériaux et géométries pour ses objets et systèmes. Ainsi, il aura une approche plutôt théorique de la performance des objets. Les illustrations 3, 4, 5 et 6 montrent le type d'objets, leur manipulation et la mise à l'épreuve que les ingénieurs peuvent réaliser avec les logiciels tels qu'Abaqus (illustrations 3 et 4) ou avec le logiciel Samcef (illustrations 5 et 6). Dans le cas des images obtenues des travaux d'un metteur-en-code du 
laboratoire, nous voyons un cube dessiné, discrétisé et soumis aux efforts. Nous pouvons observer comment les zones les plus sollicitées ont une couleur plus proche du rouge (illustration 3). Dans la figure 4 nous observons la manière dont le metteur-en-code a affiné sa discrétisation sur certaines zones de sa géométrie afin d'obtenir des résultats plus précis.

Les illustrations 5 et 6 proviennent aussi du travail d'un metteur-en-code. Les paramètres dont la plaque a été conçue dans le logiciel nous montrent un matériau composite.

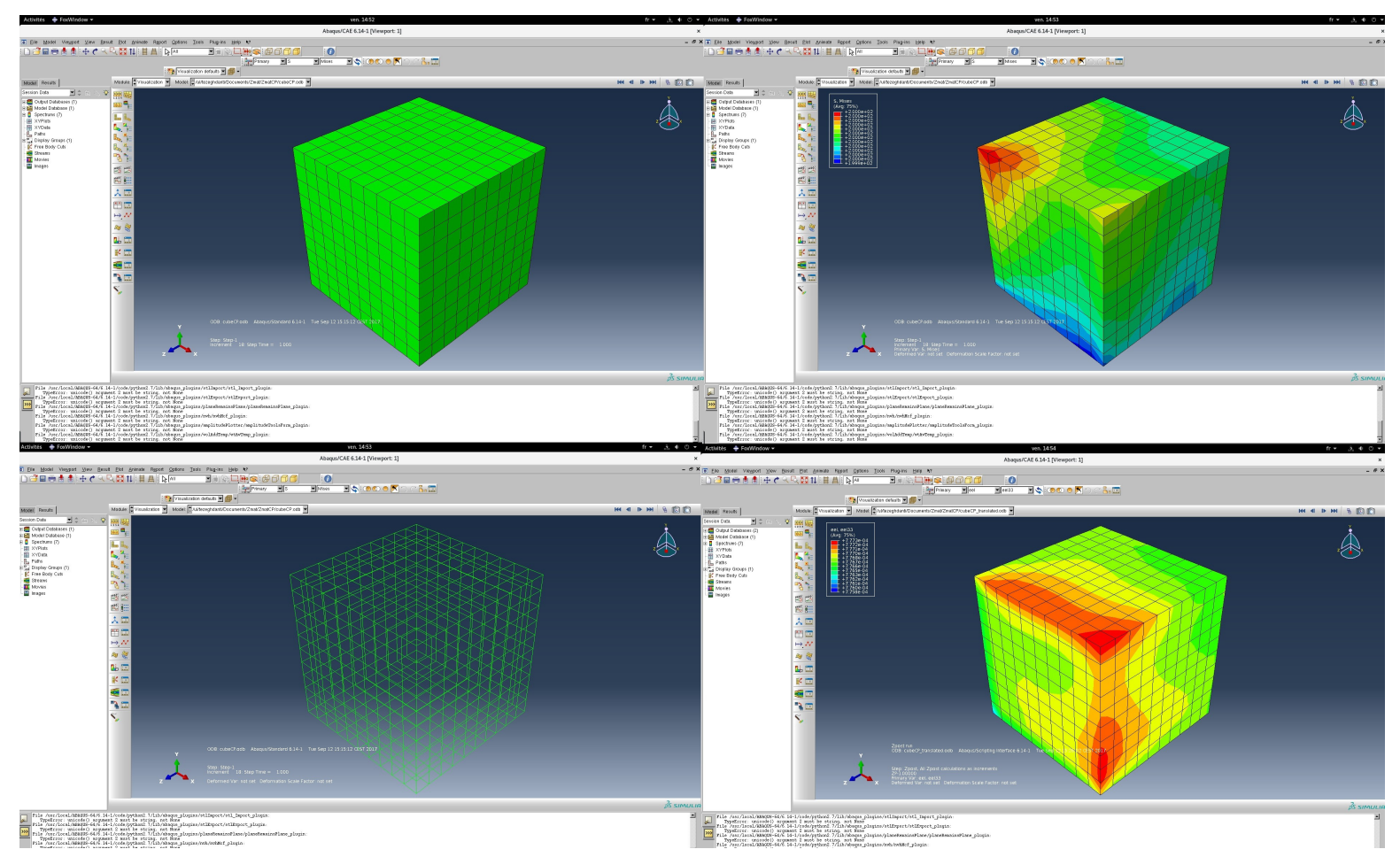

Illustration 3 : Cube dessiné, maillé et calculé avec le logiciel Abaqus 

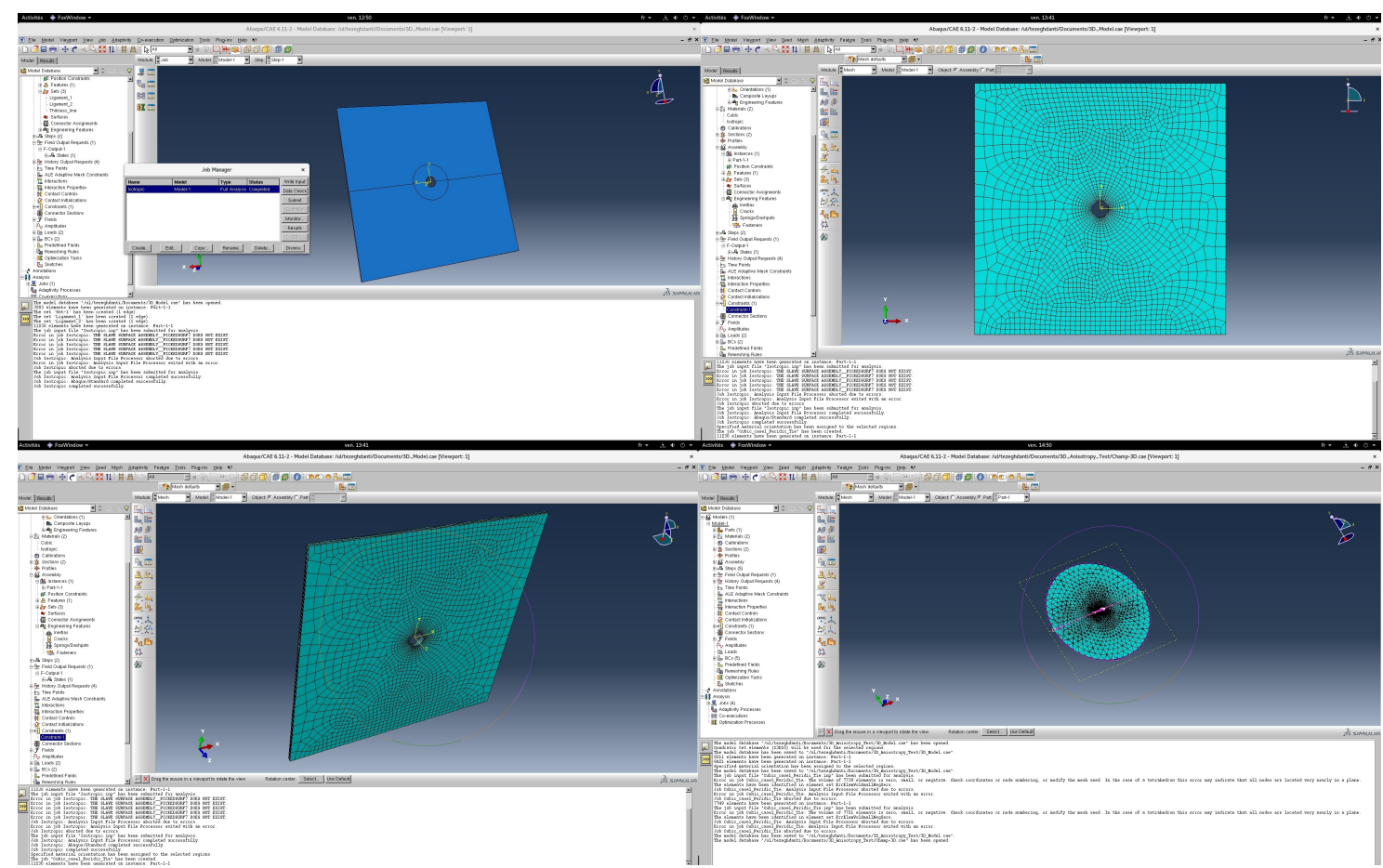

Illustration 4 : Plaque trouée dessinée et maillé sur le logiciel Abaqus
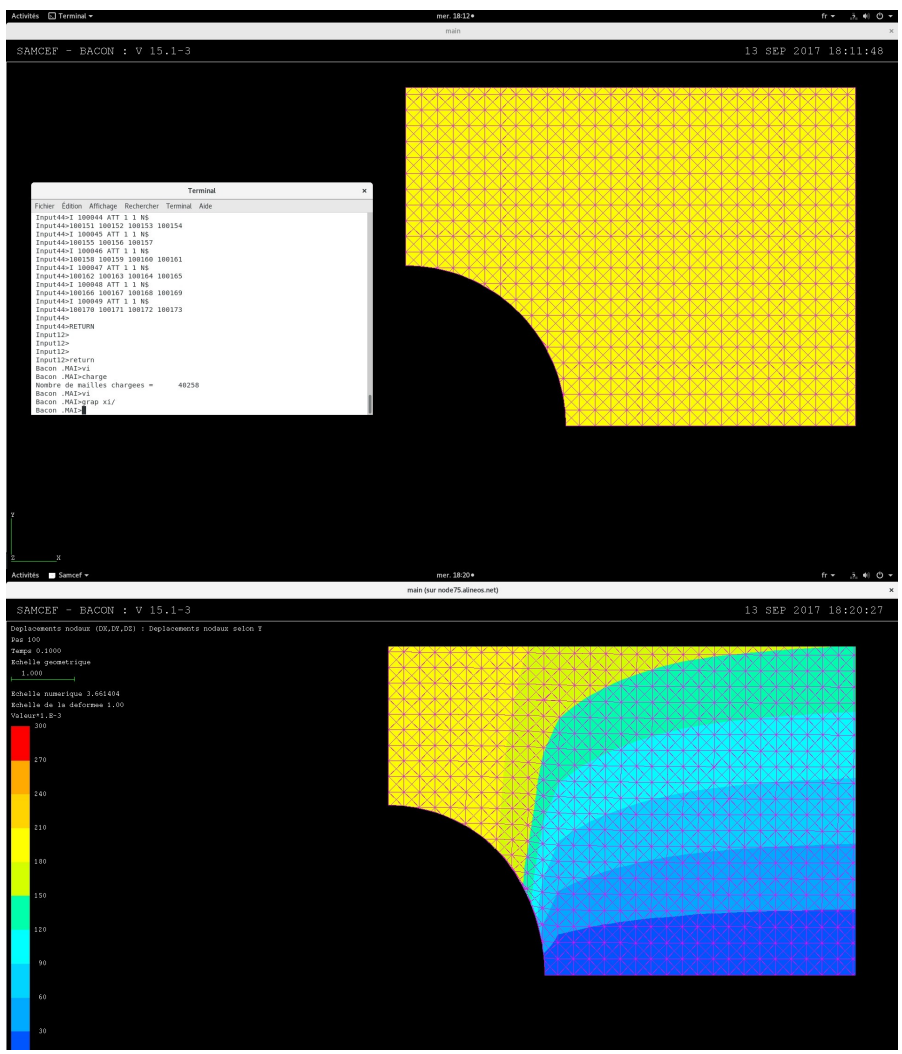

Illustration 5 : Logiciel SAMCEF, maillage et calcul EF sur une plaque trouée 


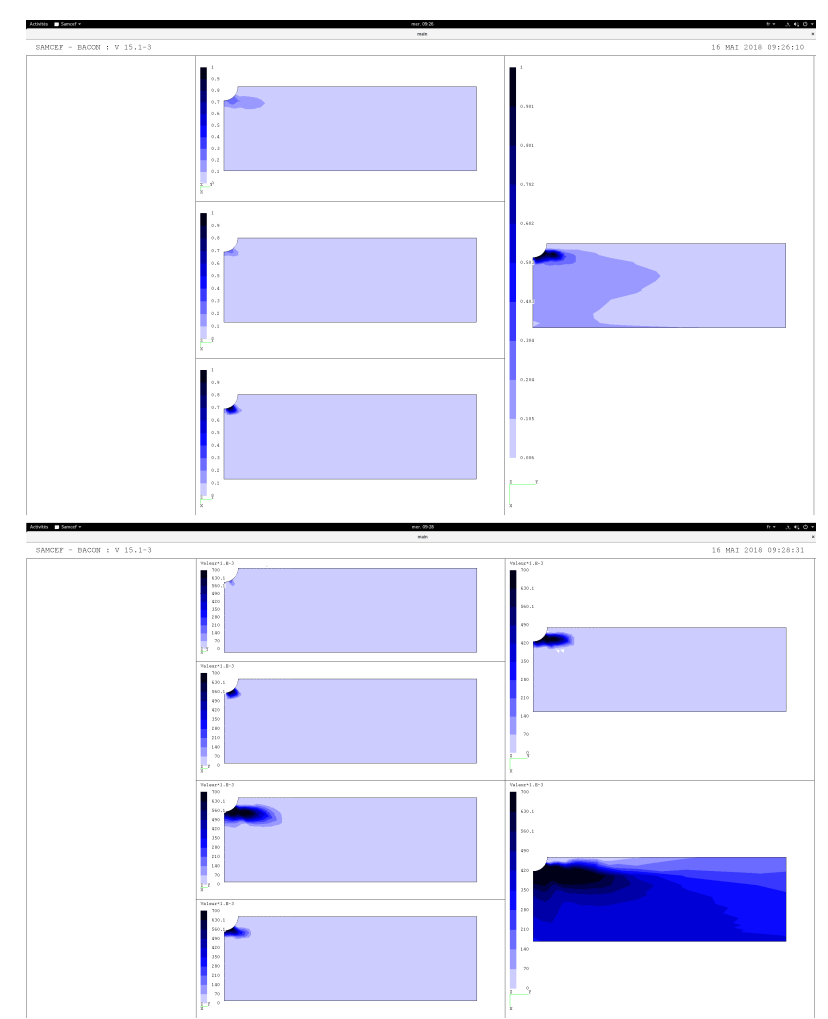

Illustration 6 : Logiciel SAMCEF, calcul d'endommagement EF, sur une plaque trouée

Les logiciels de calcul par EF sont considérés comme des outils très puissants qui permettent d'obtenir des résultats parfaitement fiables. Ce qui peut être remis en cause en revanche, c'est la pertinence des calculs des ingénieurs. Le logiciel fonctionne selon les besoins de l'ingénieur. Ainsi, les problèmes ne seront pas de l'ordre du résultat mais de l'ordre de la pertinence du calcul que l'ingénieur lance. En lien avec la pratique de l'ingénieur-calcul, nous observons le travail des dessinateurs. Ils mobilisent un autre savoir-faire et d'autres outils numériques. Ils utilisent des logiciels de conception assistée par ordinateur ou des outils CAO.

Au laboratoire étudié, les travaux des metteurs-en-code de la mécanique visent à développer des modèles sur des matériaux et des méthodes de calculs potentiellement exploitables par les ingénieurs-calculs au sein des entreprises. L'industrie aéronautique est le partenaire plus important et le plus intéressé pour le développement de ce type de recherches et de ce type d'outils pour l'ingénieur. Afin d'être utilisés dans le milieu industriel, les outils issus de la recherche doivent être introduits dans les grands logiciels, ou dans certains cas l'ingénieur pourra piloter le logiciel de calcul EF avec le programme développé par la recherche. Dans le premier cas, le processus d'introduction d'un outil de calcul dans un logiciel propriétaire 
s'appelle intégration. L'intégration est le transfert technologique entre le laboratoire et l'industrie. Ce processus est assuré intégralement par l'industrie, le laboratoire n'y participe que rarement.

« Le partenaire scientifique est exclu de ce processus de négociation, chacun son métier... on les laisse travailler, publier et après qu'ils ne nous embêtent pas quand on va faire notre business qui est faire des logiciels. On dit, « l'argent vous l'avez pour faire de la recherche. Si après vous voulez négocier avec nous pour avoir ceci cela, on dit 'non'». Chacun son métier. On paye la recherche, on vous laisse publier et après on s'occupe de l'industrialisation " (François, Ingénieur)

Mais parfois, l'intégration est à la charge du metteur-en-code qui a produit l'outil ; ce qui est intéressant car, une fois l'outil développé à l'interface du laboratoire et de l'industrie, le metteur-en-code pourra passer à l'industrie pour réaliser l'intégration. Dans le laboratoire étudié, les logiciels de calcul EF les plus utilisés sont Abaqus et Samcef. Abaqus est un des logiciels pour la simulation et l'analyse par EF le plus utilisés. Originalement produit par l'entreprise de logociel Abaqus Inc., depuis 2005 il a été racheté et ensuite produit par l'entreprise Dassault Sytsemes propriété du Groupe Industriel Marcel Dassault. Cela témoigne donc de l'intime relation entre la recherche et l'industrie des EF et les intérêts de l'industrie aéronautique. Une fois rachetée par Dassault Systems, Abaqus Inc. est devenue Simulia. Cette société développe le logiciel Abaqus et la partie « simulation » du logiciel de conception Catia. Certains doctorants du laboratoire après avoir développé un outil EF ont été embauchés par Dassault Systems afin de réaliser leur introduction dans le logiciel Abaqus. Quant à Samcef, il s'agit d'un logiciel issu du milieu académique. Il a été développé au sein du Laboratoire des Techniques Aéronautiques et Spatiales de l'Université de Liège. Cela montre aussi que la simulation et l'analyse par EF ne sont pas seulement liées à l'industrie comme dans le cas d'Abaqus, mais elles sont indissociables de la recherche universitaire dans le domaine aéronautique et spatial. En 1986, la société Samtech est créée pour commercialiser le logiciel. De nos jours, $60 \%$ des actions de la société appartiennent à une entreprise du groupe Siemens appelée LMS international. Le lien Samcef-laboratoire est étroit grâce à un contrat de partenariat. Ainsi, les chercheurs ont accès à des niveaux assez bas dans l'architecture et la programmation du logiciel. Cela représente un avantage pour la production des outils informatiques mais facilite également l'optimisation de la performance et des investissements en recherche pour les industriels. Autrement dit, cela permet de préparer et de mettre en œuvre l'intégration. Or, c'est justement l'intégration qui fait d'un outil développé dans la recherche, 
un outil d'ingénieur. Ainsi, la recherche financée par l'industriel produit des résultats dans la continuité du travail avec le laboratoire de recherche. Avec ce type de partenariat, le laboratoire prépare l'application des outils issus des coopérations, travail auparavant réalisé par industriels. Cela n'empêche pas que l'intégration reste l'affaire des industriels. D'une certaine manière, le travail de recherche autour des méthodes numériques est en lien direct avec l'industrie aéronautique mais aussi avec les éditeurs de logiciels. Ainsi, par le biais de la recherche, les entreprises du secteur aéronautique font des codes de calcul sur mesure.

Dans le système de pertinence qui relie la recherche et l'industrie dans notre cas d'analyse, la MEF permet la conformation d'un espace de communication, de production et de circulation des énoncés valables au sein duquel les positions respectives de l'industrie et de la recherche différent tout en menant des activités commentaires. Si l'ingénieur dans l'entreprise doit produire des énoncés valables, le chercheur est en charge d'élargir la possibilité de l'ingénieur de produire ces énoncés. Le chercheur et le metteur-en-code opèrent donc au niveau de la grammaire du langage (Kittler, 2008). L'ingénieur opère plutôt au niveau du sens et le chercheur au niveau de la structure du langage.

\section{Les EF et la culture du calcul numérique}

\section{Le laboratoire et l'Aéronautique, une communauté structurée en groupes de travail?}

Communauté, collectif et culture, ces trois concepts ont été au cœur des analyses sociologiques de la structure du monde scientifique. Une communauté est un groupe homogène où les différents agents se ressemblent autour de valeurs communes ; un collectif est un regroupement d'agents complémentaires (quelques-uns appartenant à une même catégorie et partageant une formation ou parcours) défini par des objectifs communs aux différentes catégories d'acteurs. Chaque catégorie d'acteurs peut aussi constituer un collectif dans un groupement majeur. Une culture se définit à travers l'ensemble de systèmes sociaux de traitement de l'information dont un groupe dispose et qu'il construit pour garantir les échanges et les relations sociales (Cicourel, 2011).

D’une certaine manière, il peut sembler paradoxal de proposer une analyse de la science, la structure la plus rationnelle de la société moderne, à travers un concept associé au monde « traditionnel », c'est-à-dire le concept de communauté. En fait, la communauté est un type spécifique de collectif qui a gouverné les modes d'analyse fonctionnaliste de la science pendant 
les années 60/70. En tant que collectif, la communauté scientifique ou le système social de la science de Merton est un système régulé par de mécanismes institutionnels (1973). En mettant l'accent sur leurs traits communs, il a souligné l'existence d'une dynamique de concurrence entre ses membres. Notamment, la communauté se reproduit en respectant les valeurs communes et en sanctionnant les transgressions. Selon Granjou et Peerbaye, Knorr-Cetina offre une vision critique des modes d'explication de l'activité scientifique selon le concept de communauté. La communauté a fonctionné comme un « collectif taxonomique » (2011:7) où les catégories du sociologue éclipseraient les dynamiques internes/propres de la recherche. Ainsi, l'idée de collectif qui émerge des critiques de la deuxième vague des études de science met en question l'image d'homogénéité que le concept de communauté transmet.

Par exemple, Michel Dubois a analysé la construction du « collectif » dans la théorie de l'acteur réseau (2007). Dubois explore les opérations théoriques de Latour et Hacking autour du développement d'une théorie de la construction de la connaissance scientifique pour comprendre les modes d'émergence des « collectifs». Comprendre la construction des faits scientifiques chez Latour consiste à analyser une dynamique collective où des agents humains et non-humains se coordonnent dans un processus de production. La construction des faits scientifiques met l'accent, en premier, sur les dynamiques de la recherche et, après, sur les produits issus des laboratoires. A partir de Latour et Hacking, Dubois identifie une dimension non-explicitée dans la construction des faits scientifiques et des collectifs de recherche. Dubois présente cette dimension à travers trois éléments : le dévoilement, la contre-factualité et la parité. Le dévoilement fait référence au fait qu'une construction présuppose un regard critique envers l'objet. La contre-factualité veut que l'objet « construit » ait une présence intimement liée à son contexte d'émergence. Le constructivisme contrefactuel efface la nécessité de l'objet scientifique des communautaristes de la première vague des études des sciences. Le constructionnisme radical met les chercheurs dans une position d'architectes qui créent un édifice selon des éléments de structure disponibles dans leur environnement de travail. De ce fait, il met sur un même plan l'objet et le sujet. Si les faits scientifiques émergent des controverses et sont le produit des négociations, pour la théorie de l'acteur réseau, les liens sociaux suivent la même dynamique. Dans cette perspective, les groupes sociaux sont le produit des controverses. En suivant, Dominique Vinck (2007), le collectif est un des produits du travail scientifique. Le collectif n'est pas un préalable mais un enjeu de l'activité de recherche. Le collectif doit être fabriqué constamment à travers les mêmes mécanismes de production des connaissances (Granjou \& Peerbaye, 2011 ; Meyer \& Molineux-Hodson, 2011). Nous avons 
constaté cette dynamique de constitution d'un collectif de travail dans notre terrain. Ce collectif se distingue par l'émergence de différents types d'acteurs, par l'introduction d'une division du travail et par l'interaction de différents modes de pratiquer la mécanique aboutissant à des produits communs. Le tournant vers la pratique introduit une approche plutôt culturaliste. En tant que " culture ", la science fonctionne comme l'unité d'un collectif hétérogène. La production des connaissances s'avère le résultat des relations entre des multiples acteurs mobilisant des différents types d'expertise. L'expertise se manifeste à travers l'ensemble de ressources matérielles et symboliques que chaque acteur a à sa disposition et mobilise dans la pratique scientifique. Le laboratoire reste l'espace privilégié des collaborations qui façonnent la production scientifique (Knorr-Cetina, 1992 ; Granjou \& Peerbaye, 2011).

\section{Recherche-industrie, les éléments constitutifs d'une culture épistémique}

Dans le cas analysé la MEF occupe la place de la grammaire permettant la constitution d'un champ de production commune. A travers la maîtrise et le partage de cette " grammaire » de la mécanique, l'activité du laboratoire avec une orientation industrielle s'organise comme un système d'actions de production des connaissances fonctionnant comme une culture épistémique (Knorr-Cetina, 1999).

D'après, la sociologue autrichienne le concept de communauté est une catégorie qui regroupe des individus selon les critères sociologiques de valeurs, d'une histoire et des symboles partagés. Pour l'étude des sciences, les analyses des co-citations reflètent le caractère communautaire de l'activité scientifique. Elle soutient aussi que partir du concept de communauté pour décrire l'activité scientifique consiste à assumer un point de départ macroscopique pour la compréhension de la structure sociale. En opposition, les représentants du constructivisme en sociologie des sciences avancent vers des questions sur le caractère de la structure sociale de la science par rapport aux relations qui se tissent entre les différents acteurs de la recherche. Knorr-Cetina (1982) va plus loin et soutient que le concept de communauté n'est pas pertinent pour l'analyse des communautés locales de travail scientifique.

Au moment de regarder la pratique scientifique quotidienne, les espaces de recherche s'avèrent le terrain d'interaction des multiples acteurs mobilisant des savoirs particuliers qui se complètent et qui entrent parfois en contradiction. La culture épistémique se base dans les modes d'échange informationnels des différents acteurs incarnant des savoirs rentrant en tension pour la production des connaissances. Dans le cas de la culture de la mécanique 
numérique la dimension épistémique acquiert une valeur importante car les modes d'appropriation des langages qui servent de base à la communication, permettent de reconnaître des positions dans une cartographie d'acteurs divers : ingénieurs, chercheurs et metteurs-encode. Le concept de culture épistémique se joue dans la multiplicité qui gouverne l'activité scientifique et qui néanmoins n'empêche pas la perception d'une unité et cohérence interne. Les différents acteurs ${ }^{3}$ raisonnent sans un horizon de communauté. Autrement dit, le raisonnement scientifique ne peut pas s'aligner au même temps avec le concept de communauté tel qu'il a été défini plus haut et les interactions des multiples agents qui rentrent en action réciproque dans les sites de recherche. La culture épistémique dépasse la communauté locale et s'enracine dans des horizons relationnels plus limités. Ainsi, les différents acteurs partagent un langage commun qu'ils explorent et exploitent différemment (comme nous l'avons vu dans la section précédente). Dans la diversité des opérations autour du langage commun, ils constituent une culture autour des EF. Les EF apportent la dimension épistémique de cette culture de la mécanique numérique.

L'image que Knorr-Cetina nous offre pour clarifier son concept de culture épistémique est celle d'un terrain où les différents agents avec des différentes fonctions semblent jouer un même jeu. Dans notre cas d'analyse autour des EF, même si les acteurs incarnent des rôles différents ${ }^{4}$, cela ne suffit pas pour affirmer qu'ils jouent des jeux différents. Néanmoins, l'unité ne vient pas du fait de partager un nombre de traits qui font apparaître une communauté. Une culture épistémique est le produit de l'évolution des différentes positions dans le jeu selon « ce qui est transmis entre les agents dans une succession de scènes continues [et] imbriquées » (KnorrCetina, 1982 : 118). Le champ scientifique apparaît ainsi dans une cartographie de relations et de flux d'échanges informationnels entre les différents agents. Une culture épistémique émerge dans la multiplicité des échanges tout comme le champ scientifique. La culture épistémique repose sur l'analyse de ce qui est transmis dans les relations entre les différents acteurs du laboratoire (Knorr-Cetina, 1999). Transmission des produits du travail contre partage des « valeurs » communes, cela est le changement que la notion de culture épistémique propose. La nature des relations a plus à voir avec un échange sur la base des ressources matérielles et symboliques que chaque agent mobilise avec une orientation vers d'autres agents. Les ressources qui circulent et qui se transforment dans la transmission entre les agents occupent

\footnotetext{
3 -techniciens, chercheurs, étudiants, personnel administratif, acteurs du monde privé, les différents types de partenaires, les autorités ministérielles ou publiques auxquels le laboratoire doit répondre-

${ }^{4}$ Les ingénieurs seront plus liés à l'exploitation des logiciels et les chercheurs/metteurs-en-code vont être plus lié à l'exploration des possibilités du langage des EF.
} 
une place centrale pour les constructivistes. Vu leur capacité à être transformées dans leur circulation, les ressources ne sont pas de choses établies mais stabilisées (Ibid.). Ainsi, elles sont constamment mises à jour dans les relations entre les agents multiples du monde de la recherche. En tant que foyer de la pratique scientifique, le laboratoire a une organisation particulière des agents qui constituent la culture épistémique et communiquent selon les langages techniques en vigueur. La question sur la communication est aussi importante pour la notion de culture épistémique. Les produits de la recherche ont une structure interne qui dépend de la grammaire qui permet leur émergence et qui reflet aussi l'accumulation des connaissances à l'intérieur d'une culture épistémique ponctuelle.

Une question qui se pose à partir des dynamiques des objets numériques et des objets techniques dans la mécanique numérique des EF, concerne l'orientation épistémique aux objets des sciences de l'ingénieur. En sciences de l'ingénieur, il « existe » ce qui a été projeté avant selon les normes de la représentation informatique (qui doit aussi converger avec les résultats expérimentaux). Dans une optique de production industrielle, le statut des objets de la mécanique informatique peut-il nous conduire à considérer les sciences de l'ingénieur dans un registre épistémique intermédiaire entre les sciences de la vie et la physique de hautes particules décrites par Knorr-Cetina ? La physique des hautes énergies se caractérise justement par le manque empirique, selon l'auteure autrichienne. Dans les expériences de ce type de physique, les objets naturels n'apparaissent que rarement. Absent dans les faits, le phénomène existe à travers ses traces dans les détecteurs ou l'ensemble des instruments de mesure, de simulation et de correction. Ce n'est pas le phénomène lui-même que les physiciens manipulent mais le jeu d'ombres, des reflets de lumière qu'il laisse dans son passage. C'est ce genre de manifestations que les détecteurs peuvent capter et les chercheurs interpréter. Ainsi, l'objet naturel de cette discipline est la déduction des images négatives du monde. Selon Knorr-Cetina, la physique de hautes énergies se caractérise par une culture épistémique négative qui opère à travers des signes de ses objets sur la base des technologies de représentation (elle n'a pas affaire avec la manipulation des objets eux-mêmes). A l'opposé, la biologie moléculaire se distingue par une culture épistémique positive. Cette science se caractérise pour la manipulation des objets pour la production de connaissance. Autrement dit, la biologie moléculaire construit les objets naturels comme des objets de la recherche. La légitimité de la connaissance dans cette science dépend du contact avec les objets. Cela rejoint les analyses de Louvel sur l'importance de la production de résultats dans le domaine des sciences de la vie. Une thèse dépend de l'obtention et de l'élaboration des résultats expérimentaux (2011). Les objets de la biologie 
moléculaire analysés se « revendiquent», dit Knorr-Cetina, comme des êtres indépendants qui interpellent le chercheur. Les expériences se développent avec les objets et non pas avec leurs traces. Il s'agit de connaissances positives qui se construisent au contact de l'objet matériel.

Ces deux cultures épistémiques, l'une négative et l'autre positive, construisent le monde de manière différente. Mais comment fonctionnent les sciences de l'ingénieur pratiquées au sein du laboratoire ? Par exemple, le mésomodèle que nous avons vu fonctionne avec l'absenceprésence des fissures entre les fibres du matériau. Dans ce modèle, les fissures existent à partir des traces, de la perte de rigidité du solide. L’ingénieur passe ainsi des fissures à des variables qui témoignent de leur existence, de leur manifestation physique à leur représentation dans des équations et des modèles.

Néanmoins, il s'agit d'un choix phénoménologique pour l'efficacité du processus de production des objets techniques et de recherche sur la matière, car en effet, la fissure est présente, et un microscope suffit pour l'identifier. Mais cela nous renverrait à un autre ordre épistémique, à une autre discipline par exemple la physique des matériaux. Ainsi, les objets des sciences de l'ingénieur oscillent entre la matérialité concrète et sa représentation comme dans le cas de la fissure. Avec les modèles et les outils de mécanique numérique, l'ingénieur interprète des signes qui vont servir pour manipuler des objets dans l'environnement informatique pour ensuite concevoir des engins et des objets techniques.

Le metteur-en-code, l'acteur d'une culture épistémique commune pour établir des équations et les résoudre numériquement

Si à l'origine, les sciences pour l'ingénieur passaient par la mise en équations des phénomènes liés à la mécanique, aux matériaux etc. (Verin, 1993), aujourd'hui s'ajoute la question de la « mise en code ». Par la double traduction des phénomènes : d'abord dans les équations, puis leur résolution par un ordinateur : la mécanique s'informatise. Pour que les équations soient résolues par un logiciel, elles doivent prendre une forme différente, la forme d'un code informatique. La mécanique passe de plus en plus par les ordinateurs. Depuis la conception jusqu'à la certification.

Au laboratoire étudié, le calcul de structures se concentre sur la question de l'endommagement des matériaux. Une des équipes du laboratoire, le « secteur structures », présente une division du travail qui nous permet de distinguer clairement ceux qui partant des problèmes industriels proposent des solutions à travers la « mise en équation » des phénomènes mécaniques et ceux 
qui mettent en place des solutions exploitables pour l'industriel. Ces derniers font la « mise en code » des solutions proposées par les metteurs-en-équations aux problèmes de la mécanique. Le partage d'une culture épistémique vient du fait que les équations doivent respecter une certaine forme afin d'être potentiellement traduites en codes EF. Inversement, les metteurs-encode maitrisent les équations au point de pouvoir les adapter au langage des ordinateurs.

La mise en équation a été présentée par Hélène Verin comme le point de départ de l'art et de la méthode des ingénieurs (1993). La matière obéit à des rapports calculables, des rapports susceptibles d'être saisis sous la forme des modèles mathématiques. Les chercheurs qui assurent la mise en équation produisent des modèles qui guident la résolution des problèmes mécaniques. Les chercheurs metteurs-en-équation visent à introduire leurs modèles dans le monde industriel. Les metteurs-en-code assurent le passage des modèles au monde industriel sous la forme de code informatique. Les metteurs-en-code sont pour la plupart des doctorants qui ont des Contrats à Durée Déterminée (CDD) d'environ 36 mois. Ce processus entre l'équation et le code ne se passe pas sans tensions entre les metteurs-en-équations et les metteurs-en-code. Il y aura des cas où de très bonnes solutions mécaniques-mathématiques proposées par les metteurs-en-équation ne seront pas intégrables simplement car l'ordinateur n'admet pas leur formulation en termes de code. Ainsi, le retour vers l'équation est possible à partir d'une impossibilité matérielle de l'ordre de l'informatique.

Nous avons dit qu'en termes de mécanique numérique, on pouvait distinguer ce qui est de l'ordre des modèles et des matériaux d'un côté et les méthodes de résolution des équations de l'autre. L'un des enjeux de la modélisation-simulation est le développement des techniques d'optimisation du milieu de la conception. Les metteurs-en-équations et leurs correspondants metteurs-en-code vont consacrer des efforts pour développer des méthodes qui ont pour but de réduire le temps de calcul pour des structures aéronautiques. Les nouveaux méthodes et modèles visent à perfectionner des environnements de conception avec l'horizon de la productivité. Dans notre cas, le lien science-industrie se base sur la confiance partagée dans la formalisation mathématique et sa représentation informatique.

Comme nous l'avons évoqué dans la section précédente, pour faire l'intégration d'un outil de calcul dans un logiciel, l'industriel aéronautique parlera avec l'éditeur du logiciel (par exemple Dassault Systems ou Samtech/Siemens), qui d'abord l'intégrera dans une version interne que l'industriel utilise Par la suite, si l'expérience est positive, il l'intégrera dans la version standard. Cela est très important car le code commercial est normé selon les normes ISO. De ce fait, il peut être utilisé pour la conception et pour la production. Selon l'autorité de certification et 
selon les normes de certification, les procédures d'obtention des valeurs sont très réglementées. Pour les calculs, il faut utiliser des outils standard, considérés comme fiables. L'autorité de certification n'acceptera jamais une pièce conçue à partir de calculs faits avec des outils non normés.

Mécanique traditionnelle/mécanique numérique, la rationalisation des pratiques de recherche et des ingénieurs à travers le numérique

La culture épistémique de la mécanique numérique se développe aussi par rapport aux façons traditionnelles de pratiquer la mécanique. Un aspect important de cette culture numérique est sa démarcation de la culture de la mécanique « traditionnelle » appelée analytique, plus ancienne et encore enseignée et pratiquée à l'université. Les générations de mécaniciens qui ont fondé le laboratoire pratiquent davantage la mécanique analytique que la mécanique numérique. Nous allons désormais montrer les points de démarcation de la culture de la mécanique numérique de celle de la mécanique « traditionnelle ».

La rationalisation des pratiques d'ingénieur conditionne la production des outils développés à l'interface du laboratoire et l'industrie aéronautique. La recherche adapte en permanence les moyens et les fins de l'action des ingénieurs. Cette opération est au cœur de la démarche industrielle et selon Max Weber, de la « modernité » (2003).

La mécanique entre la tradition et la rationalisation des pratiques de recherche et d'ingénieur à travers le numérique

Le calcul numérique reconfigure des pratiques, des choix techniques et cognitifs opposant les méthodes traditionnelles du champ [méthodes analytiques] aux méthodes propres de la mécanique informatisée.

Ces dynamiques de la tradition et d'une sorte de « modernité » liée au numérique reproduisent le sens sociologique classique octroyé à l'action rationnelle motivée par la tradition et l'action rationnelle téléologique (Weber, 2003). La mécanique traditionnelle reproduit des pratiques selon une dynamique de l'action motivée selon l'habitude ou ce qui a toujours été de la même façon. L'adjectif « traditionnel » est une forme de distinction des pratiques plus qu'un mode de description de la structure du champ de la mécanique analytique ou mécanique des solutions exactes à des problèmes « simples ». La mécanique informatisée vise à trouver des réponses à 
des problèmes plus « complexes » en assumant qu'il n'existe pas une solution exacte à ce type de questions.

Le processus de rationalisation consiste à altérer l'ordre du monde de façon à rapprocher l'action présente et l'acte projeté dans un futur «attendu ». La rationalisation rapproche l'action en cours et le produit projeté par l'acteur comme « acte» (Schutz, 1994). Sur la base du discours technique, la modernité est l'époque marquée par la course permanente de la rationalisation (Weber, 2003). La rationalisation n'est possible qu'à travers l'accumulation des connaissances qui permettent aux hommes et femmes de maîtriser le monde, ainsi que d'anticiper, prévoir, produire et orienter les phénomènes selon leurs besoins. L'intellectualisation est le processus par lequel l'accumulation systématique des expériences sous la forme de connaissances valables intersubjectivement devient une source pour la construction des règles de causalité. Selon Max Weber, la rationalisation et l'intellectualisation permanentes sont les deux traits fondamentaux, voire essentiels, de l'esprit d'époque qui gouverne le monde industriel (2003). Elles produisent le désenchantement du monde et témoignent de l'optimisme positiviste par rapport au développement technique ${ }^{5}$. La rationalisation opère sur la sphère de l'action rationnelle téléologique. Elle consiste notamment à reconfigurer ou à transformer la chaîne causale pour atteindre un but donné. A travers la mécanique informatisée, le metteur-en-code opère sur la chaîne téléologique de conception liée aussi aux nécessités de certification dans le milieu aéronautique. Cette distinction entre le traditionnel et le numérique oppose deux générations. Un metteur-en-code m'a dit, pendant une discussion sur son travail autour du calcul des structures composites, que « le métier a changé, on fait plutôt de l'informatique » et que « les patrons sont très forts avec les calculs, mais ils ne comprennent pas très bien les ordis » (Fabien, Metteur-encode). Il a déclaré que son directeur lui demandait parfois des choses que l'ordinateur «n'aime pas ». Il m'a aussi raconté qu'il a dû passer du temps à expliquer à son directeur pourquoi certaines formulations mathématiques des problèmes ne pouvaient pas être introduites dans les ordinateurs tels qu'il lui demandait. Nous pouvons analyser cette tension à deux niveaux, le premier est le générationnel mais la question semble plus complexe. Nous pouvons aussi interpréter le fait que la mise-en-équation, même si elle demande une écriture qui permet ensuite la mise en code des calculs, n'exige pas non plus une connaissance totale de ce que l'ordinateur « aime ». Cela correspond exclusivement au travail des metteurs-en-code. Un autre exemple de cette opposition entre les metteurs-en-code et les metteurs-en-équation

\footnotetext{
${ }^{5}$ Néanmoins, elles dissimulent la faiblesse de l'homme face à l'hyperpuissance de l'univers objectal qui l'entoure. Le monde de la production des objets dépasse la capacité subjective des hommes à saisir une réalité qui leur échappe sans cesse.
} 
s'est produit pendant une réunion où les doctorants présentent leurs avancées de thèse. Une doctorante exposait son travail et un professeur a remarqué « tu t'es trompée dans la formule ». En fait, elle ne s'était pas trompée, ce que le professeur croyait une erreur n'était pas une formule mathématique mais un opérateur informatique dans son code. Cela a produit des commentaires entre les doctorants pour confirmer ce qu'ils « savaient », c'est-à-dire que les professeurs sont étrangers aux « règles du code ».

Par rapport à la reconfiguration des pratiques de la mécanique, nous avons identifié deux formes d'opposition de la mécanique traditionnelle à la mécanique numérique pratiquée par les metteurs-en-code. La première fait référence au type de problèmes que les chercheurs peuvent traiter avec l'une et avec l'autre. La mécanique a traditionnellement travaillé sur des problèmes linéaires. Il s'agit de questions de recherche où les causes et les effets peuvent être formulés analytiquement. La linéarité d'un phénomène suppose que si nous augmentons les causes, les effets vont augmenter de manière proportionnelle. Ce rapport peut être décrit par une formulation mathématique. Cela veut dire que la solution peut être exprimée à travers un calcul mécanique-mathématique exact ou analytique. La mécanique numérique ne remplace pas la mécanique analytique. Elle permet d'aborder un ensemble de phénomènes dans leur complexité, notamment les phénomènes non-linéaires grâce à la puissance de calcul informatique. Les phénomènes mécaniques représentés par des courbes qui partent dans tous les sens n'ont pas d'expression analytique. L'endommagement et la plasticité sont des exemples de ce type de phénomènes. Grâce à la puissance des ordinateurs, l'ingénieur ou le chercheur est en conditions de faire une linéarisation très approximative des problèmes non linéaires. La mécanique numérique peut représenter les phénomènes qui font des courbes comme la somme des petits problèmes linéaires.

La seconde renvoie aux modes d'aborder la «complexité » avec l'une et avec l'autre. La mécanique traditionnelle permet d'aborder les phénomènes de résistance des matériaux en simplifiant les géométries et les comportements. Ce type de réduction de la complexité est l'une des conditions pour la résolution des problèmes en mécanique analytique. La réduction de la complexité en mécanique traditionnelle consiste à simplifier à travers l'introduction de ce que les ingénieurs appellent des « hypothèses restrictives ». Ces hypothèses interviennent au niveau de la géométrie ou du comportement du solide. La mécanique informatisée a permis d'affiner une partie de ces hypothèses restrictives. Cela veut dire que la prise en compte de l'incertitude se voit bouleversée grâce à la puissance de calcul des ordinateurs. 


\section{Conclusion}

La simulation numérique basée sur la méthode des EF permet d'articuler le monde industriel et l'activité du laboratoire. Il est intéressant d'observer le mode dans lequel les ingénieurs et les chercheurs reproduisent et distribuent des définitions sur les modes de relation des activités, des pratiques et des rôles pour configurer la morphologie sociale de cette culture numérique commune à la recherche et l'industrie. La question du développement des méthodes de simulation devient ainsi un enjeu majeur des sciences de l'ingénieur et du laboratoire étudié en particulier. Dans le cas étudié nous avons observé que dans une large mesure, le rapport science industrie passe par cette reconfiguration des pratiques de la recherche et l'émergence d'une catégorie de mécaniciens que nous avons identifiés comme les metteurs-en-code.

Les EF permettent la constitution d'une culture épistémique autour de la mécanique numérique. La MEF fonctionne comme un langage sur lequel se structurent les échanges du laboratoire avec l'industrie aéronautique. Elle est ainsi à la base de la communication entre ces deux mondes. La MEF opère comme une grammaire de la mécanique des solides qui fonctionne sur la base de langages informatiques (dans notre cas, matlab, $\mathrm{C}++$, Python et Fortran). Cette grammaire de la mécanique est faite de structures sur lesquelles les ingénieurs peuvent projeter des objets et systèmes possibles, qui permettront de maîtriser certains phénomènes physiques.

En tant que méthode de référence de la mécanique informatisée pour la conception des systèmes techniques, les EF permettent le passage des objets numériques aux objets réels. Les EF fonctionnent comme un système de pertinences commun aux ingénieurs et aux chercheurs sur la base de la maîtrise des langages informatiques.

La grammaire est un ensemble de structures que les individus mobilisent selon un certain nombre de règles afin de créer un ordre particulier. Ces règles sont à la fois restrictives et productives. Elles vont permettre aux acteurs, dans notre cas aux ingénieurs et aux chercheurs de retraduire la complexité de phénomènes de sorte qu'ils pourront être maîtrisés à travers des objets et des systèmes techniques. Les objets susceptibles d'exister dans la grammaire des ingénieurs et chercheurs pourront devenir des objets techniques capables de vaincre les résistances que le monde offre à leur maîtrise. Si un objet peut exister dans la grammaire des sciences et de la pratique de l'ingénieur, il pourra produire des effets sur le monde réel. Tout ce qui existe de forme légitime dans le registre de cette nouvelle grammaire de l'ingénieur pourra constituer la correction de l'objet. Sans sa formulation selon les normes de la grammaire des EF, un objet pourra difficilement exister dans le monde. De surcroît, la grammaire puissante 
guidant la pratique de l'ingénieur permettra au concepteur de valider les calculs qui font son objet technique opérant dans le monde. Sur la base de la grammaire les ingénieurs seront en mesure de produire les énoncés valables qui sont à la base de la communication.

La MEF est une méthode qui structure la pratique de l'ingénieur. La recherche en sciences de l'ingénieur telle qu'elle est pratiqué au sein du laboratoire vise à élargir les « pratiques discursives » à l'intérieur de la méthode (Nofre, Priestley \& Alberts, 2014). Les chercheurs du laboratoire analysé travaillent sur la capacité des ingénieurs à produire des énoncés valables à l'intérieur des traitements par EF. Ce sont les outils qui intègrent les logiciels commerciaux qui permettent aux ingénieurs de calculer un certain nombre de phénomènes, de traiter des comportements des matériaux, d'appliquer un certain nombre de contraintes etc. aux objets qu'ils conçoivent.

La numérisation du travail et de la science des ingénieurs introduit la notion d'objet numérique comme complémentaire de l'objet technique. L'architecture des objets numériques se réalise à travers leur formation selon les règles et les signes que l'ordinateur, que les processeurs peuvent interpréter (Hui, 2016). Le traitement des objets numériques demande une cohérence entre l'opération demandée par l'utilisateur et le langage de la machine. Dans la mécanique analytique, l'objet technique correspond formulation mathématique-mécanique. Mais dans la dialectique objet technique-objet numérique, la production d'objets exige aussi une formulation numérique. Les relations entre objets numériques et objets techniques est plus large que l'idée de représentation des objets réels dans un environnement virtuel. Toutes les simulations numériques partent de et se basent sur un phénomène réel (Jebeile, 2016 ; Giere, 2009). Les simulations sont produites selon de tests et de calculs qui doivent être vérifiés et validés. Une fois stabilisées, les simulations deviennent le point de départ du processus de conception des objets techniques.

Les ordinateurs et les codes EF sont les outils qui relient industrie et laboratoire à travers la simulation numérique. La base de la communication dans cette configuration sociale passe par la compréhension mutuelle à travers le partage d'un système de pertinence et d'un langage commun. Autrement dit, la communication est assurée à travers la maitrise de la MEF et des langages informatiques. Dans cette dynamique des rapports informatisés, les chercheurs seront en charge d'élargir les fonctions que l'ingénieur pourra réaliser en termes de simulation numérique. La recherche à travers l'action des metteurs-en-code construit des matérialités informatiques que les ingénieurs vont manipuler afin de remplir une tâche particulière pour la production d'objets et de systèmes techniques. De cette manière, nous sommes en mesure 
d'identifier dans le rapport laboratoire-industrie aéronautique un environnement culturel sur la base de systèmes sociaux de traitement de l'information. Ces systèmes sociaux se caractérisent par les modes d'échanges, de circulation, de stockage et de régimes de production d'objets qu'ils introduisent.

Ces dynamiques de recherche opposent deux générations et deux «types de mécanique », la mécanique analytique ou traditionnelle et la mécanique numérique. Le développement des outils et des MEF est à l'origine de la corrélation entre la mécanique et l'informatique, c'est-àdire entre des équations et des codes. Dans notre cas d'étude, cette corrélation entre la mécanique et l'informatique opère un ensemble de relations sociales et introduit de nouveaux acteurs. Cette corrélation va des matériaux aux équations, et des équations aux logiciels. De surcroît, à chaque matérialité (soit informatique ; soit technique) correspond un ensemble de relations sociales et d'agencements techniques. Une fois le code établi et stabilisé, il s'autonomise afin de produire un effet dans le processus de conception industriel. Les metteursen-code ne sont pas seulement à l'interface de l'informatique et de la mécanique. Ils incarnent la corrélation qui existe entre les équations et leur formulation sous la forme de code informatique.

\section{Bibliographie}

CARPENTIER Jean (2010). Cent vingt ans d'innovations en aéronautique, Paris, Hermann. CICOUREL Aaron (2011). «Processus cognitive-affectifs, interactions et structure sociale », in CLEMENT Fabrice \& Kaufmann Laurence (dir.), La sociologie cognitive, Paris, Maison des Sciences de l'Homme, pp.325-352.

DUBOIS, Michel (2007). « La construction métaphorique du collectif : dimensions implicites du prêt-à-penser constructiviste et théorie de l'acteur-réseau », L'Année sociologique, 1(.57), pp. 127-150.

GIERE Ronald (2009). "Is Computer Simulation Changing the Face of Experimentation?", Philosophical Studies, 1 (143), pp. 59-62.

GRANJOU Céline \& PEERBATE Ashveen (2011). "Sciences et collectifs », Terrains \& travaux, 18, pp.5-18.

HEINTZ Christophe (2011). « Les fondements psychiques et sociaux de la cognition distribuée » in CLEMENT Fabrice \& KAUFMANN Laurence (dir.) La sociologie cognitive, Paris, Maison des Sciences de 1'Homme, pp.277-298. 
HUTCHINS Edwin (1995) Cognition in the Wild, New Ed. Cambridge, Mass, MIT Press.

HUI Yuk (2016). On the Existence of Digital Objects, Minneapolis, University of Minnesota Press.

JEBEILE Julie (2016). "Les Simulations Sont-Elles Des Expériences Numériques ? », Dialogue : Canadian Philosophical Review/Revue Canadienne de Philosophie, 1 (55), pp.59-86.

KITTLER Friedrich (2008). "Code or, How You Can Write Something Differently" In FULLER Matthieu (dir.), Software Studies: A Lexicon, Cambridge, MIT Press, pp.4047

KNORR-CETINA Karin (1999). Epistemic cultures: how the sciences make knowledge, Cambridge, Harvard University Press.

- (1992) "The Couch, the Cathedral, and the Laboratory: On the Relationship between Experiment and Laboratory in Science" In PICKERING Andrew (dir.), Science as practice and culture, Chicago, University of Chicago Press, pp.113-138.

. (1982) "Scientific Communities or Transepistemic Arenas of Research? A Critique of Quasi-Economic Models of Science”, Social Studies of Science, 1 (12), pp.101-130.

LOUVEL Séverine (2011). Des patrons aux managers : Les laboratoires de la recherche publique depuis les années 1970, Rennes, PU Rennes.

MER Stephan (1999). «L'ingénieur-calculs au bureau d'étude » In VINCK Dominique (dir.), Ingénieurs au quotidien : ethnographie de l'activité de conception et d'innovation, Grenoble, France, Presses universitaires de Grenoble, pp.77-89.

MERTON Robert, K. (1973) The sociology of science, Chicago: Chicago University Press

MEYER Morgan \& MOLYNEUX-HODGSON Suzanne (2011). " 'Communautés épistémiques' : une notion utile pour théoriser les collectifs en sciences ?», Terrains et Travaux: Revue de Sciences Sociales, ENS Cachan, 2011, 1 (18), pp.141-154

NOFRE David, PRIESTLEY Mark \& ALBERTS Gerard (2014). "When Technology Became Language: The Origins of the Linguistic Conception of Computer Programming, 1950 1960”, Technology and Culture, 1(55), pp. 40-75.

ROBERTS John (1964). “The self-management of cultures” In GOODENOUGH Ward (dir.) Explorations in Cultural Anthropology: Essays in Honor of George Peter Murdock. New York, McGraw-Hill, pp.433-454.

SCHUTZ Alfred (1994). Le chercheur et le quoitidien, Mediriens Klincksieck, Paris.

VINCK, Dominique (2007). Sciences et société : sociologie du travail scientifique. Paris, France, A. Colin. 
VERIN Hélène (1993). La Gloire des ingénieurs. Paris, Editions Albin Michel.

WEBER Max (2003). Économie et société, tome 1 : Les Catégories de la sociologie. Paris, Pocket. 